\title{
Evaluation of tomographic reconstruction methods for small animal microCT and microPET/CT.
}

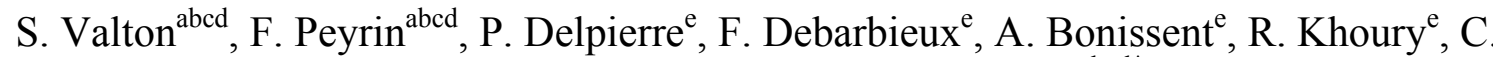 \\ Meessen $^{\mathrm{e}}$, C. Morel ${ }^{\mathrm{e}}$, D. Sappey-Marinier ${ }^{\text {abcd* }}$ \\ ${ }^{a}$ CREATIS, UMR CNRS 5515, 69621 Villeurbanne, France \\ ${ }^{b}$ CREATIS, U630 INSERM, 69621 Villeurbanne, France \\ ${ }^{c}$ CREATIS, INSA-Lyon, 69621 Villeurbanne, France \\ ${ }^{d}$ CREATIS, Université Claude Bernard-Lyon1, 69677 Bron, France \\ ${ }^{e}$ CPPM, CNRS IN2P3 UMR6550, Université de la Méditerranée-Aix-MarseilleII, 13288 Marseille, France
}

Elsevier use only: Received date here; revised date here; accepted date here

\begin{abstract}
Biological research on small animals is constantly demanding for imaging devices with higher performances like combined scanners enabling multiple modality data acquisition. In this context, we consider the concept of using hybrid X-ray pixel detectors XPAD with the ClearPET to build a prototype of microPET/CT. The system will permit simultaneous acquisition of the anatomical and functional modalities thanks to the placement of the XPAD in front of the gamma detectors. It is foreseen to place the X-ray source outside the PET ring thus involving a non-conventional off-centered circular geometry for the CT data acquisition. In a first step, a series of acquisitions was performed with a microCT built with the XPAD2 detector. Our work consists first, in the investigation of several CT reconstruction methods for these acquisitions. We hereby compare the reconstruction results obtained with two variants of the Feldkamp and two analytical methods developed to compensate for some artefacts. Second, the reconstruction issue in the off-centered geometry is addressed by testing the same algorithms on numerical simulations. It appears that, if the next generation of XPAD detectors using smaller pixels is needed to obtain high resolution images, the reconstruction method employed plays an important role and can efficiently improve the quality of the results. (C) 2001 Elsevier Science. All rights reserved.
\end{abstract}

Keywords : PET/CT, Small Animal Imaging, Cone-beam reconstruction, Circular trajectory.

\footnotetext{
" Corresponding author. Tel.: +33472436407; fax: +33472438526; e-mail: valton@creatis.insa-lyon.fr.
} 


\section{Introduction}

Medical imaging systems are steadily being improved to provide images with increased spatial resolution, sensitivity and new kinds of information. The promising potential of in vivo imaging, such as microCT and microPET, is now used as a research tool for investigating small animal models such as transgenic mice [1]. MicroCT provides high resolution anatomical images while microPET can monitor metabolic and functional mechanisms. Intensive research is therefore in progress to propose multimodal scanners adapted to small animal imaging in order to better combine the information. Based on the clinical PET/CT configuration where two different scanners are aligned on the same longitudinal axis [2], commercial systems for small animal are under development. However, to obtain combined information in a one-shot-examination, higher level of integration is needed. In this purpose, Fontaine et al. proposed new detectors enabling acquisition of both PET and CT data without bed translation [3].

In this framework, we study a new configuration of integrated system involving a full-ring PET associated with hybrid pixel detectors XPAD [5]. XPAD detectors have very high $\mathrm{X}$-rays photons counting rate while not interfering with the PET gamma rays. This feature permits their placement in front of the PET crystals while the X-ray source will rotate outside the PET ring. This particular design induces a non-standard off-centered geometry for the $\mathrm{CT}$ acquisition which requires adapted tomographic reconstruction.

Tomography consists in the reconstruction of a function from a given set of its projections along straight lines. Practically, the reconstruction from a sufficient number of projections is usually performed with analytical methods. In the case of cone-beam (CB) data acquired along a circular locus, the popular filtered back-projection (FBP) Feldkamp formula (FDK) is usually preferred for its rapidity and simplicity [6]. Nevertheless, different methods were developed to better account for the acquisition geometry for instance. The circular trajectory theoretically leads to approximate reconstruction owing to missing data except in the plane containing the X-ray source (mid-plane). The resulting reconstruction errors, referred to as $\mathrm{CB}$ artefacts, increase with the distance to the mid-plane and essentially consist in low contrast drop and geometrical deformation of the reconstructed structures. Different reconstruction algorithms which reduce these $\mathrm{CB}$ artifacts, were proposed such as the Radon-based (RB) [7] or the shift-variant filtering (SVF) [8] methods, but they require additional computation time. The choice of the reconstruction technique is a compromise between accuracy and rapidity.

For this purpose, different variants of the FDK algorithm as well as the RB and SVF methods are first tested on real acquisitions in standard circular geometry. Second as the off-centered geometry is highly exposed to $\mathrm{CB}$ artifacts, since the entire volume of interest can be outside the exactlyreconstructed mid-plane, we evaluate the same reconstruction methods on simulations in nonstandard off-centered geometry.

\section{Reconstruction of microCT acquisitions}

In a first step, a prototype of simple microCT called PIXSCAN was built using hybrid pixel detectors XPAD2 with $330 \mu \mathrm{m}$ wide pixels originally developed for crystallography [9]. Tomographic reconstruction was evaluated on real acquisitions (here: on a mouse) using the following algorithms:

- FDK: extension to $3 \mathrm{D} \mathrm{CB}$ data of the $2 \mathrm{D}$ FBP algorithm:

1. Cosine pre-weighting of the projections,

2. Ramp filtering along the detector lines,

3. CB back-projection.

The ramp filter can be associated with an apodization window, like the hanning window, to attenuate the high frequency noise hanning window.

- Radon-based (RB): sum of the FDK reconstruction and a FBP correction term corresponding to the 3D Radon transform values on the shell of the torus containing the data derived from the $\mathrm{CB}$ circular acquisition. The correction term is computed as follows:

1. Projections cosine pre-weighting, 


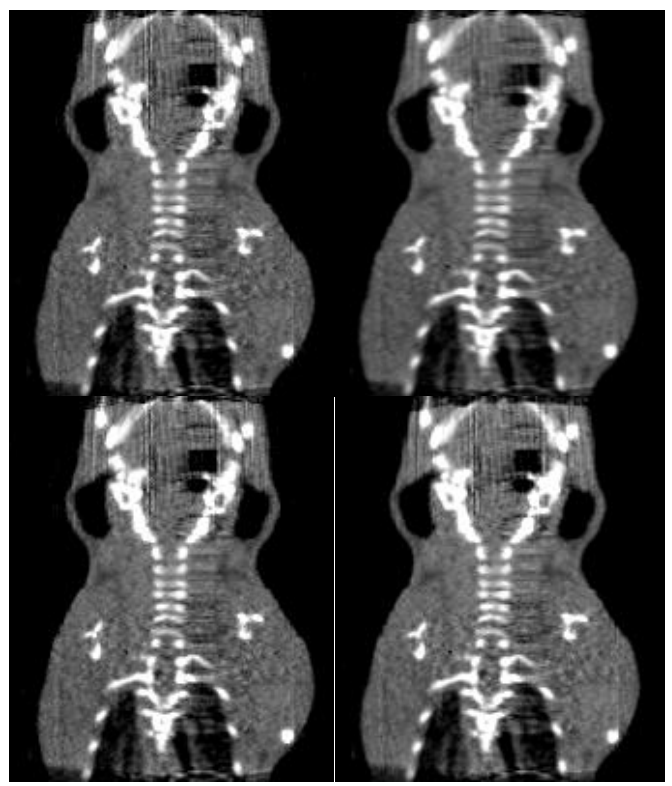

Fig.1. Mouse coronal slices reconstructed with: (top line) FDK with ramp filtering (left) and hanning window (right), and (bottom line) with RB (left) and SVF (right) methods.

2. Computation of the sum along the detector lines and derivation of this signal,

3. CB back-projection of the result.

- Shift-variant filtering (SVF): modification of the direction of the ramp filter via a reorganization of the projection data from $\mathrm{CB}$ to parallel fan-beam (PFB). The algorithm is organized as follows:

1. CB to PFB rebinning via the Fourier space,

2. Vertical rebinning for equispaced sampling,

3. SVF along the detector lines.

The reconstructions of the mouse are displayed on Fig.1. Although the resolution remains quite poor due to the rather large pixels of the XPAD2, soft tissue can be clearly distinguished from bones: lungs, spine and auditory canals can be identified on the vertical slices shown on Fig.1. Few specific artefacts appear on these images: the top and bottom of the image are strongly attenuated forming a $\mathrm{V}$ shape due to the longitudinal truncation of the object during the scan (long-object effect [10]). The vertical stripes visible in the top section of the image result from a needle located in the field of view (metal artefact [11]). The Gaussian distributed noise observed in soft tissues results mainly from the Poisson distributed noise
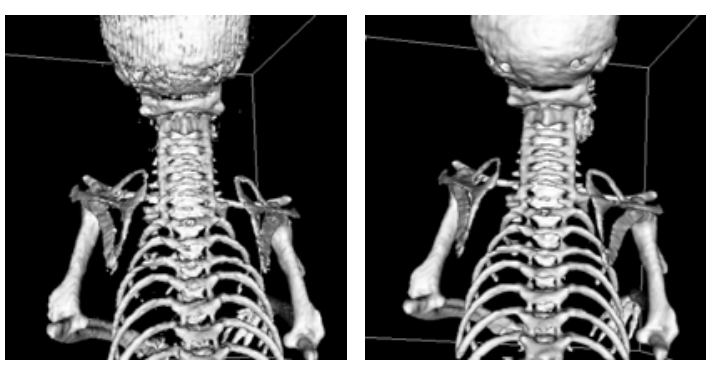

Fig.2. Surface representation of mouse skeleton from FDK reconstruction without hanning window (left) and with (right).

present on X-ray acquisitions [12]. The use of hanning apodization window in the FDK reconstruction (top right image) leads to a smoothed volume, reducing the Poisson noise as well as the beam-hardening to the detriment of spatial resolution. The extracted skeleton image thus presents smoother surfaces (Fig.2.). This can be interesting for automatic post-processing such as segmentation with level sets methods. Concerning the two CB artefacts correction methods, namely $\mathrm{RB}$ and SVF, no difference is seen on reconstructions. Actually no CB artefact is visible on the reconstructions, presumably due both to the small $\mathrm{CB}$ angle (total $\mathrm{CB}$ angle: $13^{\circ}$ ) and to the predominance of other sources of noise. Therefore, there is no point using these methods on such data since they last twice as long as the FDK without significant image improvement.

\section{Reconstruction of off-centered simulations}

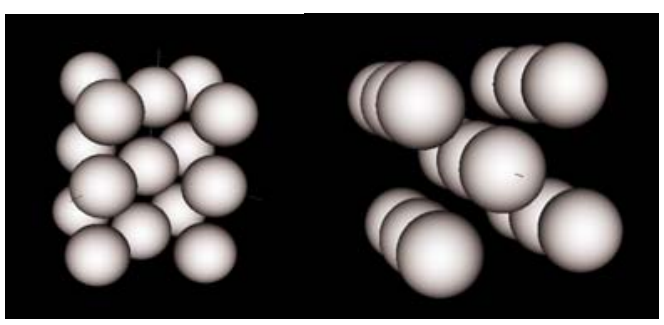

Fig.3. Perspective views of the 3D phantom used for the numerical simulations.

The concept of bimodal microPET/CT raises a challenge for the $\mathrm{CT}$ reconstruction. In a previous work, we derived from the FDK a formula called $\alpha$ FDK which accounts for the off-centered geometry 


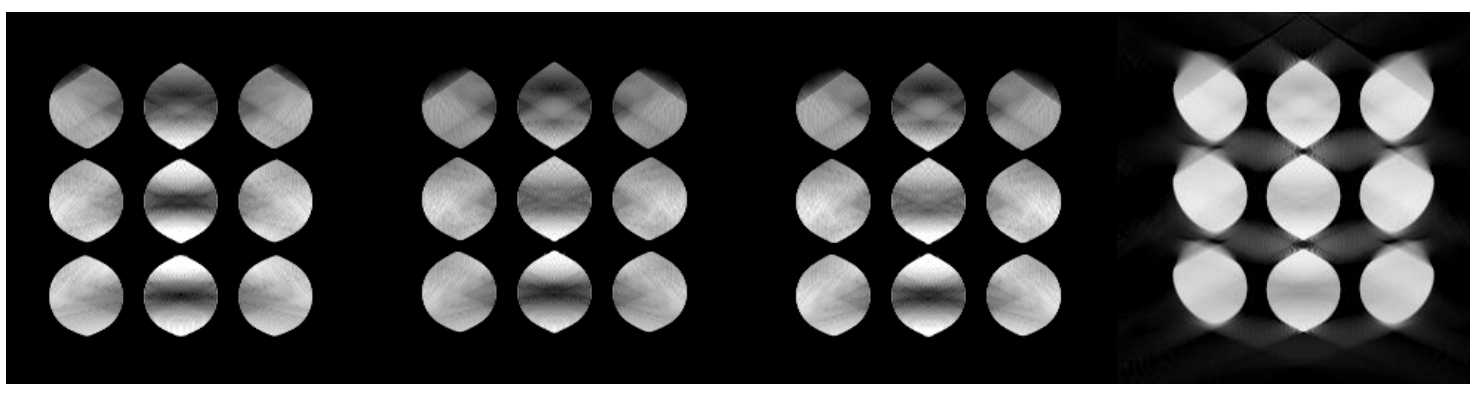

Fig.4. Phantom coronal slices reconstructed (from left to right) with unmodified FDK, $\alpha$-FDK, RB and SVF methods.

[13]. As missing data lead to severe CB artefacts, we evaluated several correction methods on simulations. The phantom used is composed of three layers of five uniform spheres forming a cross (see Fig.3).

Fig.4 displays coronal planes of volumes reconstructed with the different algorithms for an offcenter angle $\alpha$ of $28.5^{\circ}$ and the data are truncated on one side along the longitudinal axis.

We observe that the $\alpha$-FDK and the RB methods slightly compensate for the $\mathrm{CB}$ artefacts compared to the standard FDK. The SVF method gives better results in terms of reconstructed value; this is reflected by brighter grey level in the image. Nevertheless, reconstructed structures are blurred and geometrical distortions are not compensated for. This last method is thus optimal on homogeneous volumes.

\section{Conclusion}

Practical tomographic reconstruction combines the difficulties of a complex analytical inverse problem and errors added by numerous factors during the acquisition. We observed that, if the Gaussian noise is partially filtered by the use of an apodization window, another reconstruction method would be necessary to compensate for the long object effect for instance. If hardware improvements provided by the next generation of XPAD detectors will greatly improve the quality of acquired data, it should be born in mind that the choice of the reconstruction method itself can correct several kinds of artefacts.

The reconstruction in off-centered geometry was evaluated on numerical simulations to focus on the $\mathrm{CB}$ artefacts due to missing data. It was shown that the compensation methods developed for standard trajectory are not efficient enough especially in the direction of the rotation axis for the $\mathrm{CB}$ angle involved. New methods are under development to better account for this particular geometry.

The conception of new integrated system may provide better resolution and sensitivity for shorter acquisition time. However, if combined acquisition can bring new information, new challenges in image reconstruction are raised.

\section{References}

[1] D. Sappey-Marinier, O. Beuf, C. Billotey et al., Nucl. Instr. Meth. Phys. Res. A 527 (2004) 117.

[2] T. Beyer, D. W. Townsend, T. Brun et al., J. Nucl. Med. 41 (2000) 1369

[3] R. Fontaine, F Belanger, J. Cadorette et al., IEEE Trans. Nucl. Sci. 33 (2005) 691.

[4] P. Delpierre, S. Basolo, J.F. Berar et al., Nucl. Sci. Symp. Conf. Rec., Puerto Rico, 23-29 Oct. 2005, 2381.

[5] C. Morel, P. Delpierre, Nucl. Instr. Meth. Phys. Res. A (2006).

[6] L.A. Feldkamp, L.C. Davis, J.W. Kress, J. Opt. Soc. Am. 1 (1984) 612.

[7] H. Hu, Scanning 18 (1996) 572.

[8] L. Yu, X. Pan, C. A. Pelizzari, Int. J. Img. Syst. Tech 14 (2005) 213.

[9] P. Delpierre, S. Basolo, J.F. Berar et al., Nucl. Instr. Meth. Phys. Res. A (2006).

[10] M. Grass, Th. Köhler, R. Proksa, Phys. Med. Biol. 46 (2001) 1595.

[11] S. Zhao, D.D. Robeltson, G. Wang, IEEE Trans. Med. Imaging 19 (2000) 1238.

[12] T. Lei, W. Sewchand, IEEE Trans. Med. Imaging 11 (1992) 53.

[13] S. Valton, F. Peyrin, D. Sappey-Marinier, IEEE Trans. Nucl. Sci. 53 (2006) 2736. 